NEWS

\title{
By the Numbers: Coronavirus infection odds, Medicaid waivers, correlating conditions
}

\author{
BY NIKO MCCARTY
}

19 AUGUST 2021

Welcome to this month's edition of the By the Numbers Newsletter! At Spectrum, we do our best to summarize the latest autism research findings - and sometimes the best summary comes in the form of a chart or map. In this newsletter, we boil down interesting new research that is conveyed most succinctly by way of data visualizations.

As a housekeeping note, if you want to continue receiving this newsletter after today, please click here to update your preferences.

Let us know what you think of the newsletter, or tell us about your own data-rich work, at news@spectrumnews.org.

\section{Vulnerable ages for SARS-CoV-2 infection among autistic people}

People with autism age 16 and younger and those aged 40 to 60 have higher odds of being infected with SARS-CoV-2, the virus that causes COVID-19, than their non-autistic peers do, according to a new study.

The researchers analyzed data from 16,406 autistic people enrolled in Israel's largest health care organization, which provides supplemental insurance to more than half of all Israelis. The team matched each participant with a non-autistic person of the same age and gender.

Autistic people aged 16 and younger had 1.3 times higher odds of being infected with SARSCoV-2, and those in the 40 to 60 age range had double the odds, the researchers found. The results appeared in the Journal of Autism and Developmental Disorders in July. 


\section{Spectrum | Autism Research News}

https://www.spectrumnews.org

The findings suggest that people with autism or other neurodevelopmental disorders should be prioritized for the COVID-19 vaccine, according to study investigator Dana Tzur Bitan, clinical psychologist at Ariel University in the West Bank.

Israeli officials issued guidance a few weeks ago to start vaccinating children with neurodevelopmental disorders from the age of 5, Tzur Bitan says. "There is awareness towards the increased risk of morbidity among people with neurodevelopmental disorders, but I can't really connect the link and say, 'OK, they've taken our findings and turned it into a policy.'”

!function()\{"use strict";window.addEventListener("message",(function(e)\{if(void $0 !==$.data["datawrapper-height"])\{var t=document.querySelectorAll("iframe");for(var a in e.data["datawrapper-height"])for(var $r=0 ; r$ 